\title{
Effects of Weed Management Strategies and Organic Matter in Plantain (Musa paradisiaca L. ABB) Yield in Agricultural Research Farm, Afaha Nsit, Akwa Ibom State, Nigeria
}

\author{
EKPO, TUU; EKPO, NT \\ College of Education, Afaha Nsit, P.M.B, 1019, Etinan, Akwa Ibom State, Nigeria \\ *Corresponding Author Email: ekpoekpo1980@gmail.com
}

\begin{abstract}
Effects of weed infestation on plantain bunch yield in Nigeria is very alarming coupled with low nutritional status of the tropical soil. Therefore, field experiments were carried out in 2016/2017 and 2017/2018 to investigate the effects of weed management strategies and organic manure on the performance of plantain (Musa paradisiaca L.ABB). The experiments were carried out in Agricultural Education Research Farm, College of Education, Afaha Nsit. Seven treatments were replicated three times. These were sweet potato $(20,000 \mathrm{plants} / \mathrm{ha})+$ poultry manure $(20 \mathrm{t} / \mathrm{ha})$; sweet potato $(20,000$ plants/ha) + sawdust $(40 \mathrm{t} / \mathrm{ha})$; hand-slashing at three months interval + poultry manure (20t/ha); hand-slashing at three months interval + sawdust 40t/ha; sweet potato + egusi-melon + poultry manure (20t/ha); sweet potato + egusi-melon + sawdust $(40 \mathrm{t} / \mathrm{ha})$ and weedy + no organic manure. The experiment was laid out in randomized complete block design. Analysis of variance was carried out on data obtained on weed studies and plantain performance. Means were separated using Duncan Multiple Range Test at 5\% probability level. The unfertilized weedy plot reduced plantain bunch yield by $89.7 \%$ followed by $41.9 \%$ and $43.0 \%$ obtained from the sawdust treatment plots integrated with egusi-melon plus hand-slashed and sweet potato respectively. The treatment plot of sweet potato integrated with egusi-melon plus poultry manure (20t/ha) significantly controlled weeds satisfactorily, accelerated plantain growth, reduced $50 \%$ flowering time by $23.5 \%$ and significantly enhanced plantain bunch yield hence; it is recommended.
\end{abstract}

\section{DOI: https://dx.doi.org/10.4314/jasem.v25i3.24}

Copyright: Copyright (C) 2021 Ekpo and Ekpo. This is an open access article distributed under the Creative Commons Attribution License (CCL), which permits unrestricted use, distribution, and reproduction in any medium, provided the original work is properly cited.

Dates: Received: 12 December 2020; Revised: 26 January 2021; Accepted: 12 February 2021

Keywords: Plantain, sweet potato, hand-slashing, organic manure.

The dwindling food supply and population growth rate in sub-Saharan Africa are among the challenges in African continent. Government efforts geared to meet food sufficiency in Nigeria are commendable but hunger and malnutrition still remain the integral lifestyle of many citizenry. Several food crops including plantain in Nigeria can meet the desired food demands if produced in large quantity and in all year round. Plantain has the potential for poverty alleviation and provides raw materials for many industries (Agom et al., 2018). The crop has high potential to solving prevalent food shortage and scarcity since it is very prolific (IPGRI, 2002). Over 70 million people in Africa depend on plantain for food (IITA, 2009).The consumption of plantain rises tremendously among the children as fried and plantain chips, youths as roasted and plantain pepper soup, the aged class and diabetic patients consume plantain in various forms including porridge, pounded and boiled plantain. Consequently, plantain has the potential to gain unique position in African household food menu. It is the world's fourth most important staple food crop after rice, wheat, and corn (FAO, 2004). The crop is a heavy feeder and it faces serious drawbacks in production due to weed infestation and inherent poor soil fertility (Onwueme; Sinha, 1991). These constitute nuisance and challenges to plantain growers making plantain production sometimes unprofitable venture. The use of herbicide for weed control is limited because of high cost, sometimes not available, requires training for proper use and it creates health hazard. These factors make rural farmers depend almost on their traditional hand-slashing and this manual labour is unreliable, cumbersome and sometimes the labour is not timely available while it requires repeated operations and it is costly (Ekpo; Ndaeyo, 2011b). Therefore, integrated weed management using edible cover crops for weed control is presently gaining popularity in several cropping systems (Etim, et al. 2010; Ekpo, et al. 2011a). Production of plantain to meet the high demand for consumption in developing countries calls for less dependency on chemical fertilizer. Idem; Udo 
(2017) highlighted the implications of chemical fertilizer to include unsustainability, environmental pollution, leaching, probability of its availability and unaffordability by the rural farmers. The meritorious benefits of organic manure on the physicochemical properties of soils have gained prominence in renewed interest in organic farming (Adeoye, 2005). At present, sawdust is usually dumped or burnt near sawmill in Nigeria. It also adds organic matter to the soil particularly when it is properly composed (Anon, 2016).Currently, its use is not popular; little information on its performance on crop is available. On the other hand positive effects of poultry manure on plantain growth and development were reported by Ndukwe et al. (2012) and Emma-Okafor et al. (2017). Generally, poultry manure contains all the essential nutrients required for crop production (Mbah; Mbagkwu, 2003), and its value as an organic fertilizer and a source of plant nutrients has been recognized (Telkamp, 2015) but it is not used to its maximum potential due to lack of recognition of its economic value (Anon, 2016). In the light of the above, the general objective of this study was to examine the effects of weed management strategies and organic matter on plantain performance.

\section{MATERIALS AND METHODS}

Experimental site: Agricultural Education Research Farm, College of Education, AfahaNsit.

Method of soil and sawdust analyses: Prior to planting 30 core samples were collected from experimental site with auger at $0-15 \mathrm{~cm}$ depth. These were bulked together, air dried and later ground. Routine soil analysis was carried out to determine the physicochemical properties of the soil. The sawdust was also air dried and later ground before it was analyzed to determine its physicochemical properties.

Experimental design and layout: The seven treatments were laid out in randomized complete block design with three replicates.

The treatments namely: sweet potato $(20,000$ plants/ha) plus poultry manure $20 \mathrm{t} / \mathrm{ha}(\mathrm{sw}+\mathrm{p})$, sweet potato (20,000plants/ha) plus sawdust (40t/ha) (sw+sa), hand-slashing at three months interval plus poultry manure $(20 \mathrm{t} / \mathrm{ha}) \mathrm{sl}+\mathrm{p}$, hand-slashing at three months interval plus sawdust (40t/ha) sl+sa, sweet potato (20,000plants/ha) plus egusi-melon (20,000plants/ha) plus poultry manure 20t/ha $(\mathrm{sw}+\mathrm{m}+\mathrm{p})$, sweet potato $(20,000$ plants/ha) plus egusi- melon 20,000plants/ha plus sawdust 40t/ha $(\mathrm{sw}+\mathrm{m}+\mathrm{sa})$, weedy + no organic manure $(w o+m o)$.

Land preparation: In the planting season usually March, the land was cleared, the biomass and the stumped removed. Thereafter, the soil was manually tilled in April, 2017. The experimental area was demarcated according to treatments and replicates. Each treatment plot measured $420 \mathrm{~m}^{2}$ and the net plot measured $270 \mathrm{~m}^{2}$.

Planting and cultural operations: The plantain suckers and sweet potato vines were procured from seed multiplication centre, Etinan, AkwaIbom State. Plantain suckers were planted 3x2m apart in holes dug $40 \mathrm{~cm} x 40 \mathrm{~cm} x 40 \mathrm{~cm}$ in May 2017.

The plantain population (1667/ha) contained five stands per treatment plot and only three located within the centre were used for data collection. The seeds of egusi-melon were sown three per hole and spaced one metre apart. It was thinned to two per hole to maintain a population of 20,000plants/ha. The potato vines were planted three per stand and later thinned to two to maintain a density of 20,000 plants/ha. In the plot of sweet potato and egusi-melon $(\mathrm{sw}+\mathrm{m})$,egusi-melon was integrated in the plot of sweet potato in early September during the 'August' break. Hand-slashing was repeated at three months interval.

Application of organic manure: Sawdust was treated with ultracide solution to prevent termites attack. Sawdust and poultry manure were applied 40t/ha and $20 \mathrm{t} /$ ha respectively at planting of plantain using ring application method. The control plot was weedy and no manure was applied throughout the study.

Weed studies: Weed data were collected at 2, 4, 6, 8, and 10 months after planting. The weed studies included weed density and dry weed biomass.

Data collection on plantain: Data collection on plantain was undertaken at 2, 4, 6 and 8months after planting. The growth parameters were the plant height, stem girth, number of functional leaves, leaf area and leaf area index. The yield components were number of hands, fingers per bunch, the girth and length of each finger. The yield was measured in tonnes/ha.

Data analyses: Analysis of variance was used while the means were separated by Duncan multiple range test. 


\section{RESULTS AND DISCUSSION}

The soil analysis in Table 1 shows that potassium, calcium, phosphorus, and some trace nutrients were insufficient for the growth and development of plantain being a heavy feeder. The analyses of organic manure namely sawdust and poultry droppings showed that sawdust contained inadequate exchangeable cations while poultry dropping was nutritionally superior to sawdust (Table 2). Both materials are used as organic manure in crop production (Mbah; Mbagwu, 2003)

Table 1: Soil physical and chemical properties of the experimental

\begin{tabular}{|c|c|}
\hline Soil Properties & Values \\
\hline $\mathrm{pH} /: /(\mathrm{H} 2 \mathrm{O})$ & 5.1 \\
\hline Organic carbon $\mathrm{gkg}^{-1}$ & 26.0 \\
\hline Soil organic matter $\mathrm{gkg}^{-1}$ & 48.3 \\
\hline Total Nitrogen $\mathrm{gkg}^{-1}$ & 5.1 \\
\hline $\mathrm{P}\left(\mathrm{mgkg}^{-1}\right)$ & 28.6 \\
\hline \multicolumn{2}{|l|}{ Exchangeable $\left(\mathrm{molkg}^{-1}\right)$} \\
\hline $\mathrm{Ca}$ & 15.5 \\
\hline $\mathrm{Mg}$ & 1.7 \\
\hline $\mathrm{Na}$ & 0.9 \\
\hline $\mathrm{K}$ & 0.5 \\
\hline Exchangeable acidity & 0.2 \\
\hline \multicolumn{2}{|c|}{$\begin{array}{l}\text { Extractible micro nutrient (Mgkg- } \\
\left.{ }^{-}\right)\end{array}$} \\
\hline $\mathrm{Mn}$ & 180.2 \\
\hline $\mathrm{Fe}$ & 17.5 \\
\hline $\mathrm{Cu}$ & 0.9 \\
\hline $\mathrm{Zn}$ & 11.2 \\
\hline Particle size & $\left(\mathrm{kgk}^{-1}\right)$ \\
\hline Sand & 711.0 \\
\hline Silt & 199.8 \\
\hline Clay & 89.2 \\
\hline
\end{tabular}

Table 2: Sources and chemical properties of organic manure

\begin{tabular}{lll}
\hline & Poultry Manure & Sawdust \\
\hline $\mathrm{pH}$ & 8.80 & 7.80 \\
$\mathrm{E} \mathrm{c}(\mathrm{u} / \mathrm{m})$ & 1921.00 & 388.0 \\
Organic carbon \% & 31.3 & 50.01 \\
Total N & 2.39 & 0.49 \\
$\mathrm{C} / \mathrm{N}$ & 13.40 & 98.06 \\
$\mathbf{M g} / \mathbf{K g}$ & & \\
$\mathrm{Ca}$ & 13800.00 & 3121.00 \\
$\mathrm{Mg}$ & 3559.00 & 1771.00 \\
$\mathrm{~K}$ & 4200.00 & 2811.20 \\
$\mathrm{Na}$ & 2152.50 & 556.10 \\
$\mathrm{P}$ & 722.15 & 162.10 \\
\hline
\end{tabular}

Weed management Strategies: In an attempt to curb weed interference in plantain production; sweet potato, sweet potato integrated with egusi-melon; egusi-melon and hand-slashing integrated in the same treatment plot were used as weed management strategies. Egusi-melon was efficient to control the early flush of weeds within 3-4 months (Table 3 and 4). This could be attributed to its rapid growth rate and $100 \%$ ground cover and thereafter declined. The efficiency in weed suppression by egusi-melon was reported by Ekpo, et al. (2011a). The early utilization of egusi-melon for weed suppression favoured the growth of plantain as reported by Ekpo; Ekpo (2019).However, sweet potato has the potential for longer and sustainable weed control that egusi-melon; this is due to the short-lived of melon while sweet potato maintained weed suppression up 6-7 months. The effectiveness of sweet potato in weed suppression was reported by Njoku et al. (2007) and attributed this to persistent ground cover attained by the crop. The integration of sweet potato plus egusi-melon was an indication to further strengthen the prolonged weed suppression than either sweet potato or egusi-melon alone. This was favoured by the bi-modal rainfall regime by favouring two periods of planting in a year (March-April) and (August-September). The integration of weed management strategies usually provides the best and sustainable weed management as reported by Ibedu, et al. (1993). Generally, the weed management strategies used in this study enhanced the early development of plantain due to their ability to curb early weed interference. The early development of plantain uninterrupted by weed interference probably indicated its superiority over later effect of weed provided adequate and quality nutrients were available.

Plantain growth and yield: The weed management strategies that suppressed weeds in the treatment plot fertilized with sawdust produced insignificant low growth parameters, yield components and bunch yield compared with poultry manure. This could be attributed to relatively low exchangeable cations present in the sawdust as reported by Idem; Udo (2017).The application of sawdust (40t/ha) plus handslashing at three months interval reduced plantain bunch yield /ha by $48.5 \%$ (2016/2017) and $42.4 \%$ (2017/2018) compared with yield from the plot treated with poultry manure in planted and ration crops respectively. Sweet potato+sawdustreduced $50 \%$ flowering time by 7.6 and $6.8 \%$ while, slashing + sawdust reduced flowering time by 2.52 and $1.71 \%$ in planted and ration crops respectively; whereas sweet potato plus poultry manure reduced $50 \%$ flowering time by 24.4 and $21.4 \%$ in the planted and ration crops respectively. This indicated that sawdust also produced relatively low essential plant nutrients compared with poultry manure (Table 2). Okonkwo, et al. (2012) reported that physical and chemical properties are richer in sawmill dumpsite than soil from non-dumpsite; hence the soil treated with 
sawdust produced plantain growth parameters and bunch yield than the unfertilized weedy plot (control).

Table 3: Effects of weed management strategies on weed density $\left(\right.$ No.m $\left._{-}{ }^{2}\right)$ in plantain plots

\begin{tabular}{llllll}
\multicolumn{5}{c}{ Planted crop (2016/2017) } & Months after planting (MAP) \\
\hline Treatments & $\mathbf{2}$ & $\mathbf{4}$ & $\mathbf{6}$ & $\mathbf{8}$ & $\mathbf{1 0}$ \\
\hline & & & & & \\
$\mathrm{Sw}+\mathrm{p}$ & $6.8 \mathrm{~b}$ & $4.7 \mathrm{c}$ & $7.3 \mathrm{c}$ & $30.8 \mathrm{c}$ & $51.7 \mathrm{~b}$ \\
$\mathrm{Sw}+\mathrm{sa}$ & $7.0 \mathrm{~b}$ & $5.0 \mathrm{c}$ & $7.0 \mathrm{c}$ & $35.5 \mathrm{c}$ & $50.9 \mathrm{~b}$ \\
$\mathrm{M}+\mathrm{sl}+\mathrm{p}$ & $68.9 \mathrm{a}$ & $13.8 \mathrm{~b}$ & $85.7 \mathrm{~b}$ & $52.0 \mathrm{~b}$ & $45.0 \mathrm{bc}$ \\
$\mathrm{M}+\mathrm{s} 1+\mathrm{sa}$ & $72.0 \mathrm{a}$ & $14.0 \mathrm{~b}$ & $86.2 \mathrm{~b}$ & $50.9 \mathrm{~b}$ & $43.9 \mathrm{bc}$ \\
$\mathrm{Sw}+\mathrm{m}+\mathrm{p}$ & $6.5 \mathrm{~b}$ & $3.8 \mathrm{c}$ & $4.5 \mathrm{c}$ & $5.1 \mathrm{~d}$ & $27.8 \mathrm{c}$ \\
$\mathrm{Sw}+\mathrm{m}+\mathrm{sa}$ & $6.7 \mathrm{~b}$ & $3.6 \mathrm{c}$ & $4.4 \mathrm{c}$ & $4.9 \mathrm{~d}$ & $28.3 \mathrm{c}$ \\
Wo+mo & $70.1 \mathrm{a}$ & $103.2 \mathrm{a}$ & $198.3 \mathrm{a}$ & $192.7 \mathrm{a}$ & $180.1 \mathrm{a}$ \\
Ratoon crop & $(\mathbf{2 0 1 7 / 2 0 1 8 )}$ & & & & \\
Sw+p & $8.2 \mathrm{~b}$ & $5.3 \mathrm{c}$ & $12.9 \mathrm{~cd}$ & $43.8 \mathrm{c}$ & $63.3 \mathrm{~b}$ \\
$\mathrm{Sw}+\mathrm{sa}$ & $8.5 \mathrm{~b}$ & $6.1 \mathrm{c}$ & $14.0 \mathrm{~cd}$ & $42.2 \mathrm{c}$ & $62.9 \mathrm{~b}$ \\
$\mathrm{M}+\mathrm{sl}+\mathrm{p}$ & $67.9 \mathrm{a}$ & $14.0 \mathrm{~b}$ & $53.8 \mathrm{~b}$ & $52.7 \mathrm{~b}$ & $48.0 \mathrm{bc}$ \\
$\mathrm{M}+\mathrm{s}+\mathrm{sa}$ & $67.7 \mathrm{a}$ & $13.7 \mathrm{~b}$ & $55.0 \mathrm{~b}$ & $54.3 \mathrm{~b}$ & $47.5 \mathrm{bc}$ \\
$\mathrm{Sw}+\mathrm{m}+\mathrm{p}$ & $7.5 \mathrm{~b}$ & $5.8 \mathrm{c}$ & $7.5 \mathrm{c}$ & $8.0 \mathrm{~d}$ & $31.2 \mathrm{~cd}$ \\
$\mathrm{Sw}+\mathrm{m}+\mathrm{sa}$ & $7.0 \mathrm{~b}$ & $6.0 \mathrm{c}$ & $6.3 \mathrm{c}$ & $8.7 \mathrm{~d}$ & $33.0 \mathrm{~cd}$ \\
$\mathrm{Wo}+\mathrm{mo}$ & $68.7 \mathrm{a}$ & $190.5 \mathrm{a}$ & $184.2 \mathrm{a}$ & $184.5 \mathrm{a}$ & $177.8 \mathrm{a}$ \\
\hline
\end{tabular}

Means in the same column followed by the same letters are not significantly different at $p=.05($ DMRT). sw+p sweet potato 20,000 plants/ha + poultry manure $(20 t / h a) . S w+s a=$ sweet potato 20,000 plants/ha + sawdust $(40 t / h a) . M+s l+p=$ hand-slashing at 3 months interval + sawdust $(40 t / h a) . M+s l+p=h a n d-$-slashing at 3 months interval + poultry manure $(20 t / h a), S w+m+p=$ sweet potato 20,000plants/ha + egusi-melon 20,000plants/ha + poultry manure $(20 t / h a)$, wo + mo $=$ weedy and no manure.

Table 4: The effect of weed management strategies on dry weed biomass $\mathrm{g}\left(\mathrm{m}^{-1}\right)$

\begin{tabular}{|c|c|c|c|c|c|}
\hline \multicolumn{6}{|c|}{ Planted crop (2016/2017) Months after planting (MAP) } \\
\hline Treatments & 2 & 4 & 6 & $\mathbf{8}$ & 10 \\
\hline $\mathrm{Sw}+\mathrm{p}$ & $0.34 \mathrm{~b}$ & $0.11 \mathrm{c}$ & $0.24 \mathrm{~cd}$ & $1.8 \mathrm{c}$ & $5.55 \mathrm{~b}$ \\
\hline $\mathrm{Sw}+\mathrm{sa}$ & $0.36 \mathrm{~b}$ & $0.13 \mathrm{c}$ & $0.22 \mathrm{~cd}$ & $1.6 \mathrm{c}$ & $5.49 \mathrm{~b}$ \\
\hline$M+s l+p$ & $6.81 \mathrm{a}$ & $3.80 \mathrm{~b}$ & $12.07 \mathrm{~b}$ & $10.72 \mathrm{~b}$ & $4.31 \mathrm{bcd}$ \\
\hline $\mathrm{M}+\mathrm{sl}+\mathrm{sa}$ & $6.79 \mathrm{a}$ & $3.90 \mathrm{~b}$ & $12.02 \mathrm{~b}$ & $10.93 \mathrm{~b}$ & $4.10 \mathrm{bcd}$ \\
\hline $\mathrm{Sw}+\mathrm{m}+\mathrm{p}$ & $0.32 \mathrm{~b}$ & $0.13 \mathrm{c}$ & $0.13 \mathrm{cde}$ & $0.26 \mathrm{~d}$ & $2.79 \mathrm{e}$ \\
\hline $\mathrm{Sw}+\mathrm{m}+\mathrm{sa}$ & $0.32 \mathrm{~b}$ & $0.11 \mathrm{c}$ & $0.14 \mathrm{cde}$ & $0.23 \mathrm{~d}$ & $2.81 \mathrm{e}$ \\
\hline Wo+mo & $6.72 \mathrm{a}$ & $11.31 \mathrm{a}$ & $22.81 \mathrm{a}$ & $29.5 \mathrm{a}$ & $32.7 \mathrm{a}$ \\
\hline \multicolumn{6}{|c|}{ Ratoon crop $(2017 / 2018)$} \\
\hline $\mathrm{Sw}+\mathrm{p}$ & $0.42 \mathrm{~b}$ & $0.20 \mathrm{c}$ & $1.29 \mathrm{~cd}$ & $5.12 \mathrm{c}$ & $8.2 \mathrm{~b}$ \\
\hline $\mathrm{Sw}+\mathrm{sa}$ & $0.40 \mathrm{~b}$ & $0.18 \mathrm{c}$ & $1.3 \mathrm{~cd}$ & $5.10 \mathrm{c}$ & $8.1 \mathrm{~b}$ \\
\hline$M+s l+p$ & $5.38 \mathrm{a}$ & $3.15 b$ & $13.2 \mathrm{~b}$ & $8.92 b$ & $6.3 \mathrm{~cd}$ \\
\hline $\mathrm{M}+\mathrm{sl}+\mathrm{sa}$ & $5.40 \mathrm{a}$ & $3.10 \mathrm{~b}$ & $13.1 \mathrm{~b}$ & $8.89 \mathrm{~b}$ & $6.5 \mathrm{~cd}$ \\
\hline $\mathrm{Sw}+\mathrm{m}+\mathrm{p}$ & $0.41 \mathrm{~b}$ & $0.17 \mathrm{c}$ & $0.16 \mathrm{cde}$ & $0.92 \mathrm{~d}$ & $4.1 \mathrm{e}$ \\
\hline $\mathrm{Sw}+\mathrm{m}+\mathrm{sa}$ & $0.38 \mathrm{~b}$ & $0.14 \mathrm{c}$ & $0.13 \mathrm{cde}$ & $0.94 d$ & $4.2 \mathrm{e}$ \\
\hline Wo+mo & $5.30 \mathrm{a}$ & $10.9 \mathrm{a}$ & $24.83 \mathrm{a}$ & $27.5 \mathrm{a}$ & $39.3 \mathrm{a}$ \\
\hline
\end{tabular}

Means in the same column followed by the same letters are not significantly different at $p=.05(D M R T) . s w+p=$ sweet potato 20,000 plants/ha + poultry manure $(20 t / h a) . S w+s a=$ sweet potato 20,000 plants/ha + sawdust $(40 t / h a) . M+s l+p=$ hand-slashing at 3 months interval + sawdust $(40 t / h a) . M+s l+p=h a n d-$-slashing at 3 months interval + poultry manure $(20 t / h a), S w+m+p=$ sweet potato 20,000plants/ha + egusi-melon 20,000plants/ha + poultry manure $(20 t / h a)$, wo + mo $=$ weedy and no manure.

Table 5: Effect of weed management strategies and organic manure on plantain plant height $(\mathrm{cm})$

\begin{tabular}{llllll}
\hline \multicolumn{5}{c}{ Planted crop (2016/2017) } & Months after planting (MAP) \\
Treatments & $\mathbf{2}$ & $\mathbf{4}$ & $\mathbf{6}$ & $\mathbf{8}$ & $\mathbf{1 0}$ \\
\hline Sw+p & $59.8 \mathrm{a}$ & $146.7 \mathrm{a}$ & $196.6 \mathrm{a}$ & $240.3 \mathrm{a}$ & $248.7 \mathrm{a}$ \\
Sw+sa & $58.2 \mathrm{ab}$ & $142.9 \mathrm{~b}$ & $182.5 \mathrm{c}$ & $216.2 \mathrm{c}$ & $230.1 \mathrm{c}$ \\
M+sl+p & $60.0 \mathrm{a}$ & $146.5 \mathrm{a}$ & $190.0 \mathrm{~b}$ & $229.1 \mathrm{~b}$ & $238.0 \mathrm{~b}$ \\
M+sl+sa & $58.4 \mathrm{ab}$ & $143.1 \mathrm{~b}$ & $188.9 \mathrm{~b}$ & $210.6 \mathrm{~cd}$ & $224.9 \mathrm{~cd}$ \\
Sw+m+p & $60.0 \mathrm{a}$ & $147.1 \mathrm{a}$ & $179.9 \mathrm{~cd}$ & $242.0 \mathrm{a}$ & $256.7 \mathrm{a}$ \\
Sw+m+sa & $58.8 \mathrm{ab}$ & $142.9 \mathrm{~b}$ & $183.1 \mathrm{c}$ & $217.0 \mathrm{c}$ & $231.0 \mathrm{c}$ \\
Wo+mo & $56.8 \mathrm{~b}$ & $136.7 \mathrm{c}$ & $144.9 \mathrm{~d}$ & $150.0 \mathrm{e}$ & $160.5 \mathrm{e}$ \\
Ratoon crop & $(\mathbf{2 0 1 7 / 2 0 1 8})$ & & & & \\
Sw+p & $62.7 \mathrm{a}$ & $148.6 \mathrm{a}$ & $197.8 \mathrm{a}$ & $242.1 \mathrm{a}$ & $251.0 \mathrm{a}$ \\
Sw+sa & $60.9 \mathrm{ab}$ & $144.7 \mathrm{ab}$ & $186.3 \mathrm{c}$ & $219.0 \mathrm{c}$ & $233.0 \mathrm{c}$ \\
M+sl+p & $63.0 \mathrm{a}$ & $147.9 \mathrm{a}$ & $191.0 \mathrm{~b}$ & $231.8 \mathrm{~b}$ & $239.9 \mathrm{~b}$ \\
M+sl+sa & $61.0 \mathrm{ab}$ & $145.1 \mathrm{ab}$ & $180.8 \mathrm{~cd}$ & $214.1 \mathrm{~cd}$ & $228.9 \mathrm{~cd}$ \\
Sw+m+p & $63.1 \mathrm{a}$ & $149.0 \mathrm{a}$ & $198.9 \mathrm{a}$ & $244.1 \mathrm{a}$ & $252.3 \mathrm{a}$ \\
Sw+m+sa & $61.0 \mathrm{ab}$ & $145.5 \mathrm{ab}$ & $186.0 \mathrm{c}$ & $219.7 \mathrm{c}$ & $233.1 \mathrm{c}$ \\
$\mathrm{W}+\mathrm{mo}$ & $57.2 \mathrm{~b}$ & $138.1 \mathrm{c}$ & $150.3 \mathrm{~d}$ & $156.1 \mathrm{e}$ & $163.4 \mathrm{e}$ \\
\hline
\end{tabular}


Means in the same column followed by the same letters are not significantly different at $p=.05(D M R T)$. sw+p sweet potato 20,000 plants/ha + poultry manure $(20 t / h a) . S w+s a=$ sweet potato 20,000 plants/ha + sawdust $(40 t / h a) . M+s l+p=$ hand-slashing at 3 months interval + sawdust $(40 t / h a) . M+s l+p=$ hand-slashing at 3months interval + poultry manure $(20 t / h a), S w+m+p=s w e e t$ potato 20,000plants/ha + egusi-melon 20,000plants/ha + poultry manure (20t/ha), wo +mo = weedy and no manure.

Table 6: Effect of weed management strategies and organic manure on plantain leaf area

\begin{tabular}{|c|c|c|c|c|c|}
\hline \multicolumn{6}{|c|}{ Planted crop (2016/2017) Months after planting (MAP) } \\
\hline Treatments & 2 & 4 & 6 & 8 & 10 \\
\hline $\mathrm{Sw}+\mathrm{p}$ & $1.0 \mathrm{a}$ & $2.7 \mathrm{a}$ & $7.0 \mathrm{a}$ & $8.6 \mathrm{a}$ & $9.7 \mathrm{a}$ \\
\hline $\mathrm{Sw}+\mathrm{sa}$ & $0.8 \mathrm{ab}$ & $1.7 \mathrm{~b}$ & $6.5 b$ & $7.3 \mathrm{~b}$ & $8.2 \mathrm{~b}$ \\
\hline$M+s l+p$ & $1.0 \mathrm{a}$ & $2.8 \mathrm{a}$ & $7.1 \mathrm{a}$ & $7.8 \mathrm{ab}$ & $9.7 \mathrm{a}$ \\
\hline $\mathrm{M}+\mathrm{sl}+\mathrm{sa}$ & $0.8 \mathrm{ab}$ & $1.8 \mathrm{~b}$ & $6.5 \mathrm{~b}$ & $7.2 \mathrm{~b}$ & $8.2 \mathrm{~b}$ \\
\hline $\mathrm{Sw}+\mathrm{m}+\mathrm{p}$ & $1.1 \mathrm{a}$ & $2.8 \mathrm{a}$ & $7.2 \mathrm{a}$ & $8.8 \mathrm{a}$ & $9.9 \mathrm{a}$ \\
\hline $\mathrm{Sw}+\mathrm{m}+\mathrm{sa}$ & $0.8 \mathrm{ab}$ & $1.8 \mathrm{~b}$ & $6.7 \mathrm{~b}$ & $7.4 \mathrm{~b}$ & $8.3 b$ \\
\hline Wo+mo & $0.6 \mathrm{c}$ & $1.2 \mathrm{c}$ & $4.0 \mathrm{c}$ & $5.1 \mathrm{c}$ & $7.5 \mathrm{c}$ \\
\hline \multicolumn{6}{|c|}{ Ratoon crop (2017/2018) } \\
\hline \multicolumn{6}{|c|}{ Months after planting (MAP) } \\
\hline $\mathrm{Sw}+\mathrm{p}$ & $1.2 \mathrm{a}$ & $3.1 \mathrm{a}$ & $7.5 \mathrm{a}$ & $9.0 \mathrm{a}$ & $10.3 \mathrm{a}$ \\
\hline $\mathrm{Sw}+\mathrm{sa}$ & $1.0 \mathrm{ab}$ & $2.6 \mathrm{~b}$ & $6.0 \mathrm{~b}$ & $7.3 \mathrm{c}$ & $7.8 \mathrm{c}$ \\
\hline $\mathrm{M}+\mathrm{sl}+\mathrm{p}$ & $1.2 \mathrm{a}$ & $3.0 \mathrm{a}$ & $7.4 \mathrm{a}$ & $8.8 \mathrm{~b}$ & $9.2 \mathrm{~b}$ \\
\hline $\mathrm{M}+\mathrm{sl}+\mathrm{sa}$ & $1.0 \mathrm{ab}$ & $2.8 \mathrm{~b}$ & $5.9 \mathrm{~b}$ & $6.2 \mathrm{~d}$ & $7.1 \mathrm{~d}$ \\
\hline $\mathrm{Sw}+\mathrm{m}+\mathrm{p}$ & $1.2 \mathrm{a}$ & $3.1 \mathrm{a}$ & $7.6 \mathrm{a}$ & $8.9 \mathrm{a}$ & $10.3 \mathrm{a}$ \\
\hline $\mathrm{Sw}+\mathrm{m}+\mathrm{sa}$ & $1.0 \mathrm{ab}$ & $2.7 \mathrm{~b}$ & $5.8 \mathrm{~b}$ & $7.1 \mathrm{c}$ & $7.8 \mathrm{c}$ \\
\hline $\mathrm{Wo}+\mathrm{mo}$ & $0.6 \mathrm{c}$ & $1.3 \mathrm{c}$ & $4.2 \mathrm{c}$ & $4.8 \mathrm{e}$ & $5.4 \mathrm{e}$ \\
\hline
\end{tabular}

Means in the same column followed by the same letters are not significantly different at $p=.05($ DMRT). sw+p $=$ sweet potato 20,000 plants/ha + poultry manure $(20 t / h a) . S w+s a=$ sweet potato 20,000 plants/ha + sawdust $(40 t / h a) . M+s l+p=$ hand-slashing at 3 months interval + sawdust $(40 t / h a) . M+s l+p=h a n d-s l a s h i n g$ at 3 months interval + poultry manure $(20 t / h a), S w+m+p=$ sweet potato 20,000plants/ha + egusi-melon 20,000plants/ha + poultry manure $(20 t / h a), w o+m o=$ weedy and no manure.

Table 7: Effect of weed management strategies and organic manure on plantain leaf area index (LAI)

\begin{tabular}{llllll}
\hline \multicolumn{5}{c}{ Planted crop (2016/2017)Months after planting (MAP) } \\
Treatments & $\mathbf{2}$ & $\mathbf{4}$ & $\mathbf{6}$ & $\mathbf{8}$ & $\mathbf{1 0}$ \\
\hline $\mathrm{S} w+\mathrm{p}$ & $0.3 \mathrm{a}$ & $0.5 \mathrm{a}$ & $1.4 \mathrm{a}$ & $1.7 \mathrm{a}$ & $1.9 \mathrm{a}$ \\
$\mathrm{Sw}+\mathrm{sa}$ & $0.2 \mathrm{ab}$ & $0.4 \mathrm{ab}$ & $0.7 \mathrm{~b}$ & $1.1 \mathrm{c}$ & $1.4 \mathrm{c}$ \\
$\mathrm{M}+\mathrm{sl}+\mathrm{p}$ & $0.3 \mathrm{a}$ & $0.5 \mathrm{a}$ & $1.2 \mathrm{a}$ & $1.5 \mathrm{ab}$ & $1.7 \mathrm{ab}$ \\
$\mathrm{M}+\mathrm{sl}+\mathrm{sa}$ & $0.2 \mathrm{ab}$ & $0.4 \mathrm{ab}$ & $0.7 \mathrm{~b}$ & $0.9 \mathrm{~d}$ & $1.1 \mathrm{~d}$ \\
$\mathrm{Sw}+\mathrm{m}+\mathrm{p}$ & $0.3 \mathrm{a}$ & $0.5 \mathrm{a}$ & $1.4 \mathrm{a}$ & $1.7 \mathrm{a}$ & $2.0 \mathrm{a}$ \\
$\mathrm{Sw}+\mathrm{m}+\mathrm{sa}$ & $0.2 \mathrm{ab}$ & $0.4 \mathrm{ab}$ & $0.7 \mathrm{~b}$ & $1.1 \mathrm{c}$ & $1.4 \mathrm{c}$ \\
Wo+mo & $0.1 \mathrm{~b}$ & $0.3 \mathrm{~b}$ & $0.5 \mathrm{c}$ & $0.6 \mathrm{~d}$ & $0.8 \mathrm{~d}$ \\
Ratoon crop & $\mathbf{( 2 0 1 7 / 2 0 1 8 )}$ & & & \\
Months after planting (MAP) & & & \\
Sw+p & $0.4 \mathrm{a}$ & $0.7 \mathrm{a}$ & $1.6 \mathrm{a}$ & $2.0 \mathrm{a}$ & $2.3 \mathrm{a}$ \\
$\mathrm{Sw}+\mathrm{sa}$ & $0.3 \mathrm{ab}$ & $0.5 \mathrm{ab}$ & $0.8 \mathrm{~b}$ & $1.3 \mathrm{c}$ & $1.5 \mathrm{c}$ \\
$\mathrm{M}+\mathrm{sl}+\mathrm{p}$ & $0.4 \mathrm{a}$ & $0.7 \mathrm{a}$ & $1.4 \mathrm{a}$ & $1.6 \mathrm{ab}$ & $2.0 \mathrm{ab}$ \\
$\mathrm{M}+\mathrm{sl}+\mathrm{sa}$ & $0.3 \mathrm{ab}$ & $0.5 \mathrm{ab}$ & $0.8 \mathrm{~b}$ & $1.0 \mathrm{~d}$ & $1.2 \mathrm{~d}$ \\
$\mathrm{Sw}+\mathrm{m}+\mathrm{p}$ & $0.4 \mathrm{a}$ & $0.7 \mathrm{a}$ & $1.6 \mathrm{a}$ & $2.1 \mathrm{a}$ & $2.4 \mathrm{a}$ \\
$\mathrm{Sw}+\mathrm{m}+\mathrm{sa}$ & $0.3 \mathrm{ab}$ & $0.5 \mathrm{ab}$ & $0.8 \mathrm{~b}$ & $1.3 \mathrm{c}$ & $1.5 \mathrm{c}$ \\
$\mathrm{W}+\mathrm{mo}$ & $0.2 \mathrm{~b}$ & $0.3 \mathrm{~b}$ & $0.4 \mathrm{c}$ & $0.6 \mathrm{e}$ & $0.7 \mathrm{e}$ \\
\hline
\end{tabular}

Means in the same column followed by the same letters are not significantly different at $p=.05(D M R T)$. sw $+p=$ sweet potato 20,000 plants/ha + poultry manure $(20 t / h a) . S w+s a=$ sweet potato 20,000 plants/ha + sawdust $(40 t / h a) . M+s l+p=$ hand-slashing at 3 months interval + sawdust $(40 t / h a) . M+s l+p=h a n d-$ slashing at 3 months interval + poultry manure $(20 t / h a), S w+m+p=$ sweet potato 20,000plants/ha + egusi-melon 20,000plants/ha + poultry manure $(20 t / h a)$, wo + mo $=$ weedy and no manure.

Table 8: Weed management strategies and organic manure on $50 \%$ flowering of plantain.

\begin{tabular}{lll}
\hline Treatments & $\begin{array}{l}\mathbf{2 0 1 6 / 2 0 1 7} \\
\text { Planted crop }\end{array}$ & $\begin{array}{l}\mathbf{2 0 1 7 / 2 0 1 8} \\
\text { Ratoon crop }\end{array}$ \\
\hline Sw+p & $9.0 \mathrm{c}$ & $9.2 \mathrm{c}$ \\
Sw+sa & $11.0 \mathrm{~b}$ & $10.9 \mathrm{~b}$ \\
$\mathrm{M}+\mathrm{s} 1+\mathrm{p}$ & $10.2 \mathrm{bc}$ & $10.0 \mathrm{bc}$ \\
$\mathrm{M}+\mathrm{s} 1+\mathrm{sa}$ & $11.6 \mathrm{~d}$ & $11.5 \mathrm{~d}$ \\
Sw+m+p & $9.0 \mathrm{c}$ & $9.0 \mathrm{c}$ \\
Sw+m+sa & $11.3 \mathrm{~b}$ & $11.1 \mathrm{~b}$ \\
Wo+mo & $11.9 \mathrm{a}$ & $11.7 \mathrm{a}$ \\
\hline
\end{tabular}

Means in the same column followed by the same letters are not significantly different at $p=.05(D M R T)$. sw+p $=$ sweet potato 20,000 plants/ha + poultry manure $(20 t / h a) . S w+s a=$ sweet potato 20,000 plants $/$ ha + sawdust $(40 t / h a) . M+s l+p=h a n d-s l a s h i n g$ at $3 m o n t h s$ 
interval + sawdust (40t/ha). $M+s l+p=$ hand-slashing at 3 months interval + poultry manure $(20 t / h a)$, Sw $+m+p=$ sweet potato 20,000plants/ha + egusi-melon 20,000plants/ha + poultry manure (20t/ha), wo +mo = weedy and no manure.

Table 9: The weed management strategies and organic manure on plantain bunch yield components at 15 MAP.

\begin{tabular}{lllll}
\hline Treatments & $\begin{array}{l}\text { Number of } \\
\text { fingers bunch }\end{array}$ & $\begin{array}{l}\text { Finger } \\
\text { length }(\mathbf{c m})\end{array}$ & $\begin{array}{l}\text { Finger } \\
\text { girth }(\mathbf{c m})\end{array}$ & $\begin{array}{l}\text { Number of } \\
\text { hands bunch }^{-1}\end{array}$ \\
\hline Sw+p & $37.8 \mathrm{a}$ & $22.3 \mathrm{a}$ & $12.0 \mathrm{a}$ & $6.2 \mathrm{a}$ \\
$\mathrm{Sw}+\mathrm{sa}$ & $22.8 \mathrm{c}$ & $18.0 \mathrm{c}$ & $7.9 \mathrm{~b}$ & $5.3 \mathrm{~b}$ \\
$\mathrm{M}+\mathrm{sl}+\mathrm{p}$ & $31.9 \mathrm{~b}$ & $20.5 \mathrm{~b}$ & $10.8 \mathrm{ab}$ & $5.7 \mathrm{ab}$ \\
$\mathrm{M}+\mathrm{sl}+\mathrm{sa}$ & $22.7 \mathrm{c}$ & $18.9 \mathrm{c}$ & $8.0 \mathrm{~b}$ & $4.9 \mathrm{~b}$ \\
Sw+m+p & $38.0 \mathrm{a}$ & $22.5 \mathrm{a}$ & $11.9 \mathrm{a}$ & $6.3 \mathrm{a}$ \\
Sw+m+sa & $23.0 \mathrm{c}$ & $18.1 \mathrm{c}$ & $7.8 \mathrm{~b}$ & $5.2 \mathrm{~b}$ \\
Wo+mo & $13.5 \mathrm{~d}$ & $10.4 \mathrm{~d}$ & $6.0 \mathrm{c}$ & $4.7 \mathrm{c}$ \\
Ratoon crop & & & & \\
Sw+p & $38.0 \mathrm{a}$ & $25.1 \mathrm{a}$ & $11.7 \mathrm{a}$ & $5.8 \mathrm{a}$ \\
Sw+sa & $20.7 \mathrm{c}$ & $20.8 \mathrm{c}$ & $9.0 \mathrm{c}$ & $4.8 \mathrm{~b}$ \\
M+sl+p & $33.0 \mathrm{~b}$ & $22.5 \mathrm{~b}$ & $10.1 \mathrm{ab}$ & $5.0 \mathrm{ab}$ \\
M+sl+sa & $20.5 \mathrm{c}$ & $21.0 \mathrm{c}$ & $8.9 \mathrm{c}$ & $4.8 \mathrm{~b}$ \\
Sw+m+p & $38.2 \mathrm{a}$ & $25.2 \mathrm{a}$ & $11.7 \mathrm{a}$ & $6.0 \mathrm{a}$ \\
Sw+m+sa & $20.7 \mathrm{c}$ & $21.1 \mathrm{c}$ & $8.9 \mathrm{c}$ & $5.0 \mathrm{~b}$ \\
Wo+mo & $12.8 \mathrm{~d}$ & $11.0 \mathrm{~d}$ & $6.3 \mathrm{~d}$ & $4.2 \mathrm{~d}$ \\
\hline
\end{tabular}

Means in the same column followed by the same letters are not significantly different at $p=.05($ DMRT). $s w+p=$ sweet potato 20,000 plants/ha + poultry manure $(20 t / h a)$. Sw + sa $=$ sweet potato 20,000 plants/ha + sawdust $(40 t / h a) . M+s l+p=$ hand-slashing at 3 months interval + sawdust $(40 t / h a) . M+s l+p=$ hand-slashing at 3months interval + poultry manure $(20 t / h a), S w+m+p=$ sweet potato 20,000plants/ha + egusi-melon 20,000plants/ha + poultry manure $(20 t / h a)$, wo + mo $=$ weedy and no manure .

Table 10: Plantain bunch yield (t/ha).

\begin{tabular}{lll}
\hline Treatments & $\begin{array}{l}\text { Planted } \\
\text { crop } \\
\mathbf{2 0 1 6 / 2 0 1 7}\end{array}$ & $\begin{array}{l}\text { Ratoon crop } \\
\mathbf{2 0 1 7 / 2 0 1 8}\end{array}$ \\
\hline Sw+p & $8.0 \mathrm{a}$ & $7.8 \mathrm{a}$ \\
Sw+sa & $4.3 \mathrm{c}$ & $4.7 \mathrm{c}$ \\
M+sl+p & $6.8 \mathrm{~b}$ & $6.6 \mathrm{~b}$ \\
M+sl+sa & $4.5 \mathrm{c}$ & $4.8 \mathrm{c}$ \\
Sw+m+p & $8.1 \mathrm{a}$ & $7.9 \mathrm{a}$ \\
Sw+m+sa & $4.5 \mathrm{c}$ & $4.7 \mathrm{c}$ \\
Wo+mo & $0.85 \mathrm{~d}$ & $0.78 \mathrm{~d}$ \\
\hline
\end{tabular}

Means in the same column followed by the same letters are not significantly different at $p=.05(D M R T)$. sw $+p=s w e e t$ potato 20,000 plants/ha + poultry manure $(20 t / h a) . S w+s a=$ sweet potato 20,000 plants $/$ ha + sawdust $(40 t / h a) . m+s l+p=h a n d-s l a s h i n g$ at $3 \mathrm{months}$ interval + sawdust $(40 t / h a) . m+s l+p=$ hand-slashing at 3 months interval + poultry manure $(20 t / h a), \quad$ Sw $+m+p=$ sweet potato 20,000plants/ha + egusi-melon 20,000plants/ha + poultry manure (20t/ha), wo +mo = weedy and no manure.

The weedy and unfertilized treatment plot reduced plantain bunch average yield (t/ha) by $89.3 \%$ and $90.5 \%$ in planted and ration plantain crops respectively. This is ascribed to low soil fertility, weed competition for nutrients, light, water and space (Table 2). Similar findings were reported by Emma-Okafor, et al. (2017) in unfertilized plantain-cassava mixture. Poultry manure was superior in both years to sawdust in terms of plantain growth parameters particularly in cover crop treatment plot (Table 5, 6, 7 and 8), yield components (Table 9) and bunch yield (Table 10) under the same weed management strategies.

Similarly, Idem; Udo (2017) reported a significant higher seed yield of Soybean with poultry manure than with unburnt and burnt sawdust. Akata et al. (2016) also reported significant increase in the yield of two cassava varieties with increase in the rates of ash and poultry manure. This is probably due to timely released of adequate essential plant nutrients under good weed management practices as noted by EmmaOkafor et al. (2017).

Conclusion: Weed management strategies using sweet potato or sweet potato integrated with egusi-melon plus poultry manure (20t/ha) suppressed weeds effectively, enhanced plantain growth parameters and bunch yield; hence it is recommended.

Acknowledgement: We are grateful to the TETfund of the federal republic of Nigeria for the sponsorship of this project.

\section{REFERENCES}


Adeoye, GO (2005). Organic Agriculture: A review and possible adaptation for food security in Nigeria. Proceedings of $1^{\text {st }}$ National conference on organic agriculture, held at University of Agriculture, Abeokuta, Nigeria. Sept. $12^{\text {th }}-15^{\text {th }}$, 2005

Agom, DI; Okoro US, Akpaeti, AJ (2018). Determinants of productivity and production growth of plantain in Akwalbom State, Nigeria. AKSU. J. Agr. Food Sci. 1(1): 125-131.

Anonymous, 2016. Manure is an excellent fertilizer retrieved Dec. 11, 2016.

Ekpo, TUU; Ndaeyo, NU (2011). Effects of akidi(Vignaunguiculata sub spp. sesquipedalis) planting density on growth, yields and land use efficiency of akidi/cassava intercrop. Agr. Bio. J. North Ame. 2(1):1383-1389, (2011) science Huphttp://www.scihub.org/ABJNA.

Ekpo, TUU; Udosen, UU; Ndaeyo; NU, Udounang PI (2011). Determination of the optimum density and profitability of vegetable cowpea (Vignaunquiculata sub sppsesquipedalis) for weed control in cassava production. Nig. J. Agri. Food Environ.1 (7), 63-68.

Ekpo, TUU; Ekpo, NT (2019). Effects of Weed Management System and Compost Manure on Plantain yield. Appl. Sci. Environ. Manage. 23 (9): 1643-1648.

Emma-Okafor; LC; Obiefuna, JC; Iwuanyanwu, UP; Okoli, NA; Ibewuchi, II; Alagba, RA (2017). Organic manure for sustainable Productivity of plantain/cassava mixture in the tropical ultisol of south-eastern Nigeria. In proceedings of the $4^{\text {th }}$ National Annual conference Organized by crop science society of Nigeria (CSSN) held at the University of Uyo, Uyo, Nigeria September, 10-14, (2017).

FAO (2004). Food and Agriculture Organization.Agrostat data base production year book.

Ibedu, NA; Unamma, RPA; Oko, BFD (1993). On farm evaluation of integrated weed management for plantain based intercropping. Nig. J. Weed Sci. 6: 21-27.
Idem, NUA; Udoh, EI (2017). Effects of organic and inorganic fertilizers on performance of Soybean in Uyo, Akwa Ibom State. In proceedings of the $4^{\text {th }}$ National Annual Conference organized by crop science society of Nigeria at the University of Uyo, Uyo, Nigeria between Sept. 10-14, 2017.

IITA (2009). International Institute of Tropical Agriculture, Banana and Plantain production and processing. Annual bulletin.

IPGRI 2002. (International plant Genetic Research Institute, Rome). Annual Report. ISBN 92-9043530-5.

Mbah, CN; Mbagwu, J (2003). Studies on decomposition, mineralization rate and biochemical oxygen demand of organic wastes. Int'l. J. Agri. Bio. Sci. (2): 51-54.

Ndukwe, OO; Maoneke, CO; Balyen, KP; Tenkouyano, A (2012). Effects of organic and inorganic fertilizers on nutrient concentrations in plantain. Afr. J. Bio.11 (7):1651-1658.

Njoku, SC; Muoneke, CO; Okpara, DA; Agbo, FMO (2007). Effects of intercropping varieties of sweet potato and okra in an ultisol of southeastern Nigeria. Afr. J. Bio. 8: 1650-1654.

Nwagwu, FA (2004). Effects of Alternative weed management method on weed control and the performance of plantain at LAOSO south western Nig. A Thesis, Department of Agriculture and Forestry, University of Ibadan.

Okonkwo, CI; Onyibe, C; Nwite, J; Igwe, TS; Mbah, CN (2012). Physical characteristics and maize grain in an Ultisol in south eastern Nigeria for sustainable crop production; J. Agron. 4: 23-27.

Onwueme, IC; Sinha, TD (1991). Field crop production in Tropical Africa. Netherlands: CTA. Wageningen.

Telkamp, M. (2015). The straight Poop on using chicken manure as fertilizer retrieved 16 Feb. 2015. 